\title{
Generalized Riesz Sequence Space of Non-Absolute Type and Some Matrix Mapping
}

\author{
Md. Fazlur Rahman, A. B. M. Rezaul Karim \\ Department of Mathematics, Eden University College, Dhaka, Bangladesh \\ Email address: \\ dr_mf_rahman@yahoo.com (Md. F. Rahman), abmreza27@gmail.com (A. B. M. R. Karim)
}

\section{To cite this article:}

Md. Fazlur Rahman, A. B. M. Rezaul Karim. Generalized Riesz Sequence Space of Non-Absolute Type and Some Matrix Mapping. Pure and Applied Mathematics Journal. Vol. 4, No. 3, 2015, pp. 90-95. doi: 10.11648/j.pamj.20150403.15

\begin{abstract}
Recently several authors defined and studied Riesz sequence space $r^{q}(u, p)$ of non-absolute type. In this paper for some weight $\mathrm{s} \geq 0$, we define the generalized Risez sequence space $r^{q}(u, p, s)$ of non-absolute type and determine its KotheToeplitz dual. We also consider the matrix mapping $r^{q}(u, p, s)$ to $l_{\infty}$ and $r^{q}(u, p, s)$ to $\mathrm{c}$, where $l_{\infty}$ is the space of all bounded sequences and $\mathrm{c}$ is the space of all convergent sequences.
\end{abstract}

Keywords: Sequence Space, Kothe-Toeplitz Dual, Matrix Mappin

\section{Introduction}

Throughout the paper $\mathrm{N}, \square$ denote the set of positive integers and the set of all real numbers. We also denote the collection of all finite subsets of $\mathrm{N}$ by F. Let $\omega$ be the space of all sequences, real or complex; $l_{\infty}, c$ and $c_{0}$ are respectively the space of all bounded sequences, convergent sequences and null sequences. Let $p=\left(p_{k}\right)$ be a bounded sequence of strictly positive real numbers with $\sup _{k} p_{k}=H$ and $M=\max \cdot\{1, H\}$.

Then the sequence spaces $l(p)$ and $l_{\infty}(p)$ were defined by Maddox [7] (see also [5, 13]) as follows:

$$
l(p)=\left\{x=\left(x_{k}\right) \in \omega: \sum_{k=1}^{\infty}\left|x_{k}\right|^{p_{k}}<\infty\right\},
$$

with $0<p_{k} \leq H<\infty$,

$$
l_{\infty}(p)=\left\{x=\left(x_{k}\right) \in \omega: \sup _{k}\left|x_{k}\right|<\infty\right\} .
$$

which are complete spaces paranormed by $\left.g_{1}(x)=\left(\sum_{k=1}^{\infty}\left|x_{k}\right|\right)^{p_{k}}\right)^{1 / M}$ and $g_{2}(x)=\sup _{k}\left|x_{k}\right|^{p_{k} / M}$ if and only if $\inf p_{k}>0$.
We shall assume throughout that $p_{k}^{-1}+t_{k}^{-1}=1$ provided $1<\inf p_{k} \leq H<\infty$.

In [15] Stieglitz and Tietz defined

$$
\begin{aligned}
& c s=\left\{x:\left(\sum_{i=1}^{n} x_{i}\right) \in c\right\} \\
& c_{0} s=\left\{x:\left(\sum_{i=1}^{n} x_{i}\right) \in c_{0}\right\} \\
& b s=\left\{x:\left(\sum_{i=1}^{n} x_{i}\right) \in l_{\infty}\right\} .
\end{aligned}
$$

Let $q=\left(q_{k}\right)$ be a sequence of positive real numbers and let us write

$$
Q_{n}=\sum_{k=1}^{n} q_{k}
$$

for $n \in \mathrm{N}$. Then the matrix $R^{q}=\left(r_{n k}^{q}\right)$ of the Riesz mean $\left(R, q_{n}\right)$ is given by

$$
\left(r_{n k}^{q}\right)=\left\{\begin{array}{lll}
\frac{q_{k}}{Q_{n}} & \text { if } & 0 \leq k \leq n \\
0 & \text { if } & k>n .
\end{array}\right.
$$


The Riesz mean $\left(\mathrm{R}, q_{n}\right)$ is regular if and only if $Q_{n} \rightarrow \infty$ as $n \rightarrow \infty$ see (Peterson [4, p.10], [11], [12], [14], [17], [18])

In a recent paper Sheikh and Ganie [16] defined and studied the Riesz sequence space $r^{q}(u, p)$ of non-absolute type by

$$
r^{q}(u, p)=\left\{x=\left(x_{k}\right) \in \omega: \sum_{n=1}^{\infty}\left|\frac{1}{Q_{n}} \sum_{k=1}^{n} u_{k} q_{k} x_{k}\right|^{p_{k}}<\infty\right\},
$$

where

$$
0<p_{k} \leq H<\infty .
$$

The main purpose of this paper is to define the generalized Riesz sequence space $r^{q}(u, p, s)$.We determine the KotheToeplitz dual of $r^{q}(u, p, s)$ and then consider the matrix mapping $r^{q}(u, p, s)$ to $l_{\infty}$ and $r^{q}(u, p, s)$ to $\mathrm{c}$.

In [2] Bulut and Cakar defined and studied the sequence space $l(p, s)$ and in [3] Khan and Khan defined and investigated the Cesaro sequence space $\operatorname{ces}(\mathrm{p}, \mathrm{s})$. In the same vein we define the generalized Riesz sequence space $r^{q}(u, p, s)$ in the following way.

Definition. For $s \geq 0$ we define

$$
r^{q}(u, p, s)=\left\{x=\left(x_{k}\right) \in \omega: \sum_{k=1}^{\infty}\left|\frac{1}{Q_{n}^{s+1}} \sum_{k=1}^{n} u_{k} q_{k} x_{k}\right|^{p_{k}}<\infty\right\} .
$$

If $\mathrm{s}=0$ then $r^{q}(u, p, s)$ reduces to $r^{q}(u, p)$, which is defined and studied in [16].

Define the sequence $y=\left(y_{k}\right)$ by

$$
y_{k}=\frac{1}{Q_{k}^{s+1}} \sum_{j=1}^{k} u_{j} q_{j} x_{j}
$$

Let $\mathrm{X}$ and $\mathrm{Y}$ be two subsets of $\omega$. Let $A=\left(a_{n k}\right)$ be an infinite matrix of real or complex numbers $a_{n k}$, where $n, k \in$ $\mathrm{N}$. Then the matrix A defines the A- transformation from $\mathrm{X}$ into $\mathrm{Y}$, if for every sequence $x=\left(x_{k}\right) \in X$, the sequence $A x=\left((A x)_{n}\right)$, the A-transform of $\mathrm{x}$ exists and is in $\mathrm{Y}$, where

$$
(A x)_{n}=\sum_{k} a_{n k} x_{k}
$$

For simplicity in notation, here and what follows, the summation without limits runs from 0 to $\infty$. By $(X, Y)$, we denote the class of all such matrices. A sequence $\mathrm{x}$ is to be Asummable to $l$ if $A x$ converges to $l$, which is called as the A-limit of $x$.

We mention the following inequality (see[6,9]) which will be used later. For any integer $E>1$ and any two complex numbers $a$ and $b$ have

$$
|\mathrm{a} \mathrm{b}| \leq E\left(|a|^{t} E^{-t}+|b|^{p}\right)
$$

Where $P>1$ and $p^{-1}+t^{-1}=1$.

Theorem 1.1. $r^{q}(u, p, s)$ is a complete linear metric space paranormed by $\mathrm{g}$ defined by

$$
g(x)=\left(\sum_{n=1}^{\infty}\left|\frac{1}{Q_{n}^{s+1}} \sum_{k=1}^{n} u_{k} q_{k} x_{k}\right|^{p_{k}}\right)^{1 / M}
$$

With $0<p_{k} \leq H<\infty$ and $H=\sup _{k} p_{k}, M=\max \{1, H\}$.

Proof. The linearity of $r^{q}(u, p, s)$ with respect to the coordinate wise addition and scalar multiplication follows from the inequalities which are satisfied for $x, y \in r^{q}(u, p, s)$ (see $[6, p .30])$

$$
\left(\sum_{n=1}^{\infty} \mid \frac{1}{Q_{n}^{s+1}} \sum_{k=1}^{n} u_{k} q_{k}\left(x_{k}+\left.y_{k}\right|^{p_{k}}\right)^{1 / M}\right.
$$

$\leq\left(\sum_{n=1}^{\infty}\left|\frac{1}{Q_{n}^{s+1}} \sum_{k=1}^{n} u_{k} q_{k} x_{k}\right|^{p_{k}}\right)^{1 / M}+\left(\sum_{n=1}^{\infty}\left|\frac{1}{Q_{n}^{s+1}} \sum_{k=1}^{n} u_{k} q_{k} y_{k}\right|^{p_{k}}\right)^{1 / M}$

and for any $\alpha \in \square$ (see [8])

$$
|\alpha|^{p_{k}} \leq \max \left\{1,|\alpha|^{M}\right\}
$$

It is clear that $g(\theta)=0$, where $\theta=(0,0,0, \ldots \ldots \ldots)$ and $g(x)=g(-x)$ for all $x \in r^{q}(u, p, s)$. The inequality (4) and (5) together gives the subadditivity of g and $g(\alpha x) \leq \max \{1,|\alpha|\} g(x)$.

Consider any sequence $\left(x^{i}\right)$ of points of $r^{q}(u, p, s)$ such that $g\left(x^{i}-x\right) \rightarrow 0$ and a sequence $\left(\alpha_{i}\right)$ of scalars such that $\alpha_{i} \rightarrow \alpha$. Then $\left(g\left(x^{i}\right)\right)$ is bounded, since by subadditivity the inequality

$$
g\left(x^{i}\right) \leq g(x)+g\left(x^{i}-x\right)
$$

holds. Thus we have,

$$
g\left(\alpha_{i} x^{i}-\alpha x\right)=\left[\sum_{n=1}^{\infty}\left|\frac{1}{Q_{n}^{s+1}} \sum_{k=1}^{n} u_{k} q_{k}\left(\alpha_{i} x_{k}^{i}-\alpha x_{k}\right)\right|^{p_{k}}\right]^{1 / M}
$$

$\leq\left|\alpha_{i}-\alpha\right| g\left(x^{i}\right)+|\alpha| g\left(x^{i}-x\right) \rightarrow 0$ as $n \rightarrow \infty$.

Thus the scalar multiplication is continuous. Hence $\mathrm{g}$ is a paranorm on the space $r^{q}(u, p, s)$.

It is quite routine to show that $r^{q}(u, p, s)$ is a metric space with the metric $d(x, y)=g(x-y)$ provided that $x, y \in r^{q}(u, p, s)$, where $\mathrm{g}$ is defined by (3); and using a similar method to that in [9] one can show that $r^{q}(u, p, s)$ is complete under the metric mentioned above . 


\section{Kothe-Toeplitz Duals}

If $\mathrm{X}$ is a sequence space we define [13]

$$
X^{\alpha}=\left\{a=\left(a_{k}\right): \sum_{k}\left|a_{k} x_{k}\right|<\infty, \text { for every } x \in X\right\}
$$

$X^{\beta}=\left\{a=\left(a_{k}\right): \sum_{k} a_{k} x_{k}\right.$ is convergent, for every $\left.x \in X\right\}$

$$
X^{\gamma}=\left\{a=\left(a_{k}\right): \sup _{n}\left|\sum_{k} a_{k} x_{k}\right|<\infty, \text { for every } x \in X\right\}
$$

$X^{\alpha}, X^{\beta}$ and $X^{\gamma}$ are called the $\alpha$ - (or Kothe-Toeplitz), $\beta$ - (or generalized Kothe-Toeplitz) and $\gamma$ - dual spaces of X, respectively. Note that $X^{\alpha} \subset X^{\beta} \subset X^{\gamma}$.

In this section we shall obtain the $\alpha$ -,$\beta$ - and $\gamma$-dual of $r^{q}(u, p, s)$. For our purpose we need the following lemma.

Lemma 2.1 ([10, Theorem 5.10$]$ ). (i) Let $1<p_{k} \leq H<\infty$ for every $k \in \mathrm{N}$. Then $A \in\left(l(p), l_{1}\right)$ if and only if there exists an integer $E>1$ such that

$$
\begin{array}{r}
\sup _{N \in F} \sum_{k=1}^{\infty}\left|\sum_{n \in N} a_{n k} E^{-1}\right|^{t_{k}}<\infty . \\
\text { hold and } \lim _{n} a_{n k}=\beta_{k} \text { for } k \in \mathrm{N} \\
\text { Theorem } 2.1 . \text { Let } 1<p_{k} \leq H \\
\text { the sets } D_{1}(u, p, s) \text { and } D_{2}(u, p, s) \\
D_{1}(u, p, s)=\bigcup_{E>1}\left\{a=\left(a_{k}\right) \in \omega: \sup _{n \in F} \sum_{k}\left|\sum_{n \in N}(-1)^{n-k} \frac{a_{n}}{u_{n} q_{n}} Q_{k}^{s+1} E^{-1}\right|^{t_{k}}<\infty\right\}
\end{array}
$$

and

$$
D_{2}(u, p, s)=\bigcup_{E>1}\left\{a=\left(a_{k}\right) \in \omega: \sum_{k}\left|\Delta\left(\frac{a_{k}}{u_{k} q_{k}}\right) Q_{k}^{s+1} E^{-1}\right|^{t_{k}}<\infty \text { and }\left(\left(\frac{a_{k}}{u_{k} q_{k}} Q_{k}^{s+1} E^{-1}\right)^{t_{k}}\right) \in l_{\infty}\right\} .
$$

Then

$$
\left[r^{q}(u, p, s)\right]^{\alpha}=D_{1}(u, p, s) \text {, and }\left[r^{q}(u, p, s)\right]^{\beta}=\left[r^{q}(u, p, s)\right]^{\gamma}=D_{2}(u, p, s) \text {. }
$$

Proof. Let $a=\left(a_{k}\right) \in \omega$. Then by (1) one can easily derive that

$$
a_{n} x_{n}=\sum_{k=n-1}^{n}(-1)^{n--k} \frac{a_{n}}{u_{n} q_{n}} Q_{k}^{s+1} y_{k}=\sum_{k=1}^{\infty} b_{n k} y_{k}
$$

where $n, k \in \mathbf{N}$ and

$$
b_{n k}=\left\{\begin{array}{l}
(-1)^{n-k} \frac{a_{n}}{u_{n} q_{n}} Q_{k}^{s+1}, \text { if } n-1 \leq k \leq n \\
0, \quad \text { if } 0 \leq k<n-1 \text { or } k>n
\end{array}\right.
$$

Let $B=\left(b_{n k}\right)$. Then by combining (8) with (i) of Lemma (ii) Let $0<p_{k} \leq 1$ for every $k \in \mathrm{N}$. Then $A \in\left(l(p), l_{1}\right)$ if and only if

$$
\sup _{\mathrm{N} \in F} \sup _{k}\left|\sum_{n \in N} a_{n k} E^{-1}\right|^{p_{k}}<\infty .
$$

Lemma 2.2 ([1, Theorem 6]). (i) Let $1<p_{k} \leq H<\infty$ for every $k \in \mathrm{N}$. Then $A \in\left(l(p), l_{\infty}\right)$ if and only if there exists an integer $E>1$ such that

$$
\sup _{n}\left|\sum_{n \in N} a_{n k} E^{-1}\right|^{t_{k}}<\infty
$$

(ii) Let $0<p_{k} \leq 1$ for every $k \in \mathrm{N}$. Then $A \in\left(l(p), l_{\infty}\right)$ if and only if

$$
\sup _{n, k}\left|a_{n k}\right|^{p_{k}}<\infty
$$

Lemma 2.3 ([1, Theorem 1]). Let $0<p_{k} \leq H<\infty$ for every $k \in \mathrm{N}$. Then $A \in(l(p), c)$ if and only if (6) and (7) hold and $\lim _{n} a_{n k}=\beta_{k}$ for $k \in \mathrm{N}$ also holds.

Theorem 2.1. Let $1<p_{k} \leq H<\infty$ for every $k \in \mathrm{N}$. Define the sets $D_{1}(u, p, s)$ and $D_{2}(u, p, s)$ as follows:
2.1 we see that $a x=\left(a_{n} x_{n}\right) \in l_{1} \quad$ whenever $x=\left(x_{n}\right) \in r^{q}(u, p, s)$ if and only if $B y \in l_{1}$ wheneve $y \in l(p)$.

This shows that. $\left[r^{q}(u, p, s)\right]^{\alpha}=D_{1}(u, p, s)$

Again, by Abel's transformation, we have

$$
\begin{aligned}
\sum_{k=1}^{n} a_{k} x_{k} & =\sum_{k=1}^{n-1} \Delta\left(\frac{a_{k}}{u_{k} q_{k}}\right) Q_{k}^{s+1} y_{k}+\frac{a_{n}}{u_{n} q_{n}} Q_{n}^{s+1} y_{n} \\
& =\sum_{k=1}^{\infty} c_{n k} y_{k}, \text { for } k \in \mathrm{N}
\end{aligned}
$$

where $C=\left(c_{n k}\right)$ is define as 


$$
c_{n k}=\left\{\begin{array}{lll}
\Delta\left(\frac{a_{k}}{u_{k} q_{k}}\right) Q_{k}^{s+1}, & \text { if } & 1 \leq k \leq n-1 \\
\frac{a_{n}}{u_{n} q_{n}} Q_{n}^{s+1}, & \text { if } & k=n \\
0, & \text { if } & k>n
\end{array}\right.
$$

where $n, k \in \mathrm{N}$. Thus from Lemma 2.3 with (9) we have $a x=\left(a_{n} x_{n}\right) \in c s$ whenever $x=\left(x_{n}\right) \in r^{q}(u, p, s)$ if and only if $C y \in c$ whenever $y \in l(p)$. Hence from (6) we derive that
$\sum_{k=1}^{\infty}\left|\Delta\left(\frac{a_{k}}{u_{k} q_{k}}\right) Q_{k}^{s+1} E^{-1}\right|^{t_{k}}<\infty \quad$ and $\sup _{k \in N}\left|\frac{a_{k}}{u_{k} q_{k}} Q_{k}^{s+1} E^{-1}\right|^{t_{k}}<\infty$

which shows that $\left[r^{q}(u, p, s)\right]^{\beta}=D_{2}(u, p, s)$.

Also, from Lemma 2.2 together with (9) we have $a x=\left(a_{k} x_{k}\right) \in b s$ whenever $x=\left(x_{n}\right) \in r^{q}(u, p, s)$ if and only if $C y \in l_{\infty}$ whenever $y=\left(y_{k}\right) \in l(p)$. This again gives the condition (10) which means that $\left[r^{q}(u, p, s)\right]^{\gamma}=D_{2}(u, p, s)$.

Theorem 2.2. Let $0<p_{k} \leq 1$ for every $k \in \mathrm{N}$. Define $D_{3}(u, p, s)$ and $D_{4}(u, p, s)$ as

$$
D_{3}(u, p, s)=\left\{a=\left(a_{k}\right) \in \omega: \sup _{N \in F} \sup _{k}\left|\sum_{n \in N}(-1)^{n-k} \frac{a_{n}}{u_{n} q_{n}} Q_{k}^{s+1} E^{-1}\right|^{p_{k}}<\infty\right\}
$$

and

$$
D_{4}(u, p, s)=\left\{a=\left(a_{k}\right) \in \omega: \sup _{k}\left|\Delta\left(\frac{a_{k}}{u_{k} q_{k}}\right) Q_{k}^{s+1}\right|^{p_{k}}<\infty \quad \text { and } \sup _{k}\left|\frac{a_{k}}{u_{k} q_{k}} Q_{k}^{s+1}\right|^{p_{k}}<\infty\right\} .
$$

Then

$$
\left[r^{q}(u, p, s)\right]^{\alpha}=D_{3}(u, p, s) \text { and }\left[r^{q}(u, p, s)\right]^{\beta}=\left[r^{q}(u, p, s)\right]^{\gamma}=D_{4}(u, p, s)
$$

Proof. The proof is similar as that of above theorem 2.1 by using second parts of Lemma 2.1, 2.2, and 2.3 instead of first parts, and so we omit the details.

\section{Matrix Mapping on the Set $r^{q}(u, p, s)$}

In this section we characterize the class of matrices $\left(r^{q}(u, p, s), l_{\infty}\right)$ and $\left(r^{q}(u, p, s), c\right)$.

Theorem 3.1. (i) Let $1<p_{k} \leq H<\infty$ for every $k \in \mathrm{N}$. Then $A \in\left(r^{q}(u, p, s), l_{\infty}\right)$ if and only if there exists an integer $\mathrm{E}>1$ such that

$$
\begin{array}{r}
U(E)=\sup _{n} \sum_{k}\left|\Delta\left(\frac{a_{n k}}{u_{k} q_{k}}\right) Q_{k}^{s+1} E^{-1}\right|^{t_{k}}<\infty \\
\left(\left(\frac{a_{n k}}{u_{k} q_{k}}\right) Q_{k}^{s+1} E^{-1}\right)^{t_{k}} \in l_{\infty} \text { for } n \in \mathbf{N}
\end{array}
$$

(ii) Let $0<p_{k} \leq 1$ for every $k \in \mathbf{N}$. Then $A \in\left(r^{q}(u, p, s), l_{\infty}\right)$ if and only if

$$
\sup _{n}\left|\Delta\left(\frac{a_{n k}}{u_{k} q_{k}}\right) Q_{k}^{s+1}\right|^{p_{k}}<\infty
$$

Proof. (i). Necessity. Let $A \in\left(r^{q}(u, p, s), l_{\infty}\right)$. Then
$A_{n}(x)=\sum_{k} a_{n k} x_{k}$ exists for $x \in r^{q}(u, p, s)$ and this implies that $\left(a_{n k}\right)_{k \in N} \in\left[r^{q}(u, p, s)\right]^{\beta}$ for every fixed $n \in \mathrm{N}$. So by theorem 2.1 the necessities of (11) and (12) hold.

Sufficiency. Suppose the conditions (11) and (12) hold.

For $m, n \in \mathrm{N}$, consider the equation

$$
\sum_{k=1}^{m} a_{n k} x_{k}=\sum_{k=1}^{m-1} \Delta\left(\frac{a_{n k}}{u_{k} q_{k}}\right) Q_{k}^{s+1} y_{k}+\frac{a_{n m}}{u_{m} q_{m}} Q_{m}^{s+1} y_{m}
$$

When $m \rightarrow \infty$ then from (14) we have

$$
\sum_{k=1}^{\infty} a_{n k} x_{k}=\sum_{k=1}^{\infty} \Delta\left(\frac{a_{n k}}{u_{k} q_{k}}\right) Q_{k}^{s+1} y_{k}
$$

Using inequality (2) we have from (15)

$$
\begin{gathered}
\sup _{n}\left|\sum_{k} a_{n k} x_{k}\right| \leq \sup _{n} \sum_{k}\left|\Delta\left(\frac{a_{n k}}{u_{k} q_{k}}\right) Q_{k}^{s+1}\right|\left|y_{k}\right| \\
\leq \sup _{n}\left[\left|\sum_{k} \Delta\left(\frac{a_{n k}}{u_{k} q_{k}}\right) Q_{k}^{s+1}\right|^{t_{k}} E^{-t_{k}}+\sum_{k}\left|y_{k}\right|^{p_{k}}\right] \\
\leq E\left[U(E)+g_{1}^{M}(y)\right]<\infty .
\end{gathered}
$$

This shows that $A \in\left(r^{q}(u, p, s), l_{\infty}\right)$.

(ii) The proof of second part is similar as that of part (i) 
and so omitted.

Theorem 3.2. (i) Let $1<p_{k} \leq H<\infty$ for every $k \in \mathrm{N}$. Then $A \in\left(r^{q}(u, p, s), c\right)$ if and only if (11), (12) and (13) hold and there is a sequence $\left(\alpha_{k}\right)$ of scalars such that

$$
\lim _{n} \Delta\left(\frac{a_{n k}-\alpha_{k}}{u_{k} q_{k}}\right) Q_{k}^{s+1}=0 \text { for all } k \in \mathrm{N} .
$$

Proof. Necessity. Suppose that $A \in\left(r^{q}(u, p, s), c\right)$ and 1 $<p_{k} \leq H<\infty$. Since $c \subset l_{\infty}$, so by above theorem the necessities of (11) and (12) hold. For the necessity of condition (16), we take for each fixed $\mathrm{k}$, a sequence $x^{(k)}=\left(x_{n}^{(k)}(q)\right)$ in $r^{q}(u, p, s)$ with

$$
x_{n}^{(k)}(q)=\left\{\begin{array}{lll}
(-1)^{n-k} \frac{Q_{k}^{s+1}}{u_{n} q_{n}}, & \text { if } & k \leq n \leq k+1 \\
0, & \text { if } \quad 0 \leq n<k \text { or } n>k+1 .
\end{array}\right.
$$

Then for each $k \in \mathrm{N}$ we have $A x^{(k)} \in c$, which shows that $\left(\Delta\left(\frac{a_{n k}}{u_{k} q_{k}}\right) Q_{k}^{s+1}\right)_{n \in N} \in c$. This proves the necessity of the condition (16).

Sufficiency. Suppose that the conditions (11), (12), (14) and (16) hold. Then for $x \in r^{q}(u, p, s)$, we have $\left(a_{n k}\right) \in\left[r^{q}(u, p, s)\right]^{\beta}$ for each $\mathrm{n}$ and so $A x=\sum_{k} a_{n k} x_{k}$ exists.

For every $m, n \in \mathrm{N}$, we have

$$
\sum_{k=1}^{m}\left|\Delta\left(\frac{a_{n k}}{u_{k} q_{k}}\right) Q_{k}^{s+1} E^{-1}\right|^{p_{k}} \leq \sup _{n} \sum_{k=1}^{\infty}\left|\Delta\left(\frac{a_{n k}}{u_{k} q_{k}}\right) Q_{k}^{s+1} E^{-1}\right|^{p_{k}}
$$

Letting $m, n \rightarrow \infty$ together with (11) and (16) gives

$$
\sum_{k=1}^{\infty}\left|\Delta\left(\frac{\alpha_{k}}{u_{k} q_{k}}\right) Q_{k}^{s+1} E^{-1}\right|^{p_{k}}<\infty .
$$

Also by letting $n \rightarrow \infty$ we have from (12) that

$$
\left(\left(\frac{a_{n k}}{u_{k} q_{k}} Q_{k}^{s+1} E^{-1}\right)^{p_{k}}\right) \in l_{\infty}
$$

which leads together with (17) that $\left(\alpha_{k}\right) \in D_{2}(u, p, s)$. Thus the series $\sum_{k} \alpha_{k} x_{k}$ converges for every $x \in r^{q}(u, p, s)$. Writing $a_{n k}-\alpha_{k}$ for $a_{n k}$ we have from (15).

$$
\sum_{k}\left(a_{n k}-\alpha_{k}\right) x_{k}=\sum_{k} \Delta\left(\frac{a_{n k}-\alpha_{k}}{u_{k} q_{k}}\right) Q_{k}^{s+1} y_{k}, \text { for } n \in \mathrm{N}
$$

Comparing this with Lemma 2.3 with $\beta_{k}=0$ for all $k \in \mathrm{N}$, we have the matrix $\left(\Delta\left(\frac{a_{n k}-\alpha_{k}}{u_{k} q_{k}}\right) Q_{k}^{s+1}\right)_{n, k \in N}$ belongs to the class $\left(l(p), c_{\circ}\right)$.

Thus by (18) we have

$$
\lim _{n} \sum_{k}\left(a_{n k}-\alpha_{k}\right) x_{k}=0 .
$$

Now by combining (19) with the above results one can see that $A x \in c$.

Thus the proof is complete.

If $\alpha_{k}=0$ for each $k \in \mathrm{N}$, then we have the following corollary.

Corollary 3.1. Let $1<p_{k} \leq H<\infty$ for each $k \in \mathrm{N}$. Then $A \in\left(r^{q}\left(u, p, s, c_{\circ}\right)\right)$ if and only if the conditions (11), (12) and (13) hold, and (16) also holds with $\alpha_{k}=0$ for each $k \in$ $\mathrm{N}$.

\section{References}

[1] C. G. Lascarides and I. J. Maddox, Matrix transformations between some classes of sequences, Proc. Cambridge Philos. Soc., 68(1970), 99-104.

[2] E. Bulut and O. Cakar, The sequence space $l(p, s)$ and related matrix transformations, communication de la faculatedes sciences d L'universite D'Ankara Tome, 28(1979), $33-44$.

[3] F.M. Khan and M.A. Khan, The sequence space $c e s(p, s)$ and related matrix transformations, Research Ser.Mat., 21(1991); 95-104.

[4] G.M. Petersen, Regular matrix transformations, Mc Graw-Hill publishing Co. Ltd., London-New York-Toronto, Ont., 1966.

[5] H. Nakano, Modulared sequence spaces, Proc. Japan Acad., 27(1951), 508-512.

[6] I. J. Maddox, Elements of functional analysis, Cambridge University Press, Cambridge, second edition, 1988.

[7] I. J. Maddox, Spaces of strongly summable sequences, Quart. J. Math. Oxford Ser., (2), 18 (1967), 345-355.

[8] I.J. Maddox, Paranormed sequence spaces generated by infinite matrices, Proc. Cambridge Philo. Soc., 64(1968), 335340 .

[9] I. J. Maddox, Continuous and Kothe-Toeplitz dual of certain sequence spaces, Proc.Camb.Phil.Soc., 65(1969), 431-435.

[10] K. G. Grosse-Erdmann, Matrix transformations between the sequence spaces of Maddox, J. Math.,Aual.appl., 180(1)(1993), 223-238.

[11] L. N. Mishra, V. N. Mishra and V. Sonavane, Trigonometric approximation of functions belonging to Lipschitz class by matrix operator of conjugate series of Fourier series, Advances in Difference Equations, a Springer Journal,(2013),2013:127. 
[12] L. N. Mishra, V. N. Mishra, K. Khatri, Deepmala, On The Trigonometric approximation of signals belonging to generalized weighted Lipschitz class by matrix operator of conjugate series of its Fourier series, Applied Mathematics and Computation,(Elsevier Journal), Vol.237 (2014) 252-263.

[13] Mursaleen, Matrix transformations between the new sequence spaces, Houston J. Math., 9(4)(1993),505-509.

[14] M. L. Mittal, V.N. Mishra, Approximation of signals (functions) belonging to the weighted class by almost matrix summability method of its Fourier series, International J. of Math. Sci. \& Engg. Appls. (IJMSEA) Vol. 2 No.IV (2008), 285-294.

[15] M. Stieglitz and Tietz, Math. Z., 154(1977),1-16.
[16] N. A. Sheikh and A B H Ganie, "A new paranormed sequence space and some matrix transformations", Acta Math. Acad. Paedagogicae Nyiregyhaiensis, 28(2012),47-58.

[17] V.N. Mishra, K. Khatri, L.N. Mishra and Deepmala, Trigonometric approximation of periodic signals belonging to generalized weighted Lipschitz class by Norlund-Euler operator of conjugate series of its Fourier series. Journal of Classical analysis, Vol-5, 2(2014), 91-105.

[18] V.N. Mishra, H.H. Khan, I.A. Khan, L.N. Mishra, On the degree of approximation of signals of Lipschitz class by almost Riesz mans of its Fourier series, Journal of Classical analysis, Vol-4, Number 1(2014), 79-87. 